\title{
Elaboration of Statistics Learning Objects for Mobile Devices
}

\author{
http://dx.doi.org/10.3991/ijim.v6i2.1971 \\ F.J. Tapia-Moreno, H.A.Villa-Martinez, and C.A. Lopez-Miranda \\ University of Sonora, Hermosillo, México
}

\begin{abstract}
Mobile learning (m-learning) allows a person to study using a mobile computer device anywhere and anytime. In this work we report the elaboration of learning objects for the teaching of introductory statistics using cellular phones.
\end{abstract}

Index Terms-electronic learning, mobile computing, statistics.

\section{INTRODUCTION}

In the University of Sonora, the undergraduate students in Mathematics, Physics, and Computer Science take a required course in introductory statistics. The course aims to teach students basic statistical tools and familiarize them with the statistical analysis using statistical software. These software tools run only on desktop computers or on laptops, which means that students must be either in the computer lab or carrying their laptops.

Moreover, considering that in 2009 there were nearly 80 million cellular subscribers in Mexico ${ }^{1}$ and that globally, about $50 \%$ of cell phones are enabled for Java ${ }^{2}$, we are interested in whether mobile phones can used to solve statistical problems, helping students to learn statistics at any time, any place and releasing them from having to carry a laptop or having to be in the computer lab.

Thus, in February 2010 the project Statistics-to-Go was proposed to the Division of Natural Sciences at the University of Sonora with the aim of producing tools for teaching statistics for cellular phones using Java ME (Micro Edition). Java ME was chosen because almost all mid- and high-range cellular phones are Java-enabled, which means that programs written in Java ME, known as "MIDlets", are portable across different mobile operating systems.

In this paper we present the major advances that have been generated in the project Statistics-to-Go in its first one and half year.

The remainder of this paper is composed as follows: Section II presents the definition of mobile learning and its main advantages over other types of learning. Section III details Java ME, and what can be created on mobile devices using this language. Section IV shows the results of the project-to-Go Statistics. Section V discusses the limitations of this work. Finally, Section VI presents our conclusions and future work.

${ }^{1}$ http://www.elsemanario.com.mx/news/news_display.php?story_id=234 88. Last visit: 13/Sept./ 2011

${ }^{2}$ http://uclue.com/?xq=1180. Last visit: 13/Sept./ 2011.

\section{MOBILE LEARNING}

Mobile learning (or m-learning) is the acquisition of knowledge through a mobile computing technology like cellular phones and personal digital assistants (PDAs). Mlearning allows a person to learn anytime and anywhere. We believe that in the near future, this type of learning will be used on a daily basis by self-taught people.

The differences between m-learning and other types of learning can be studied from two perspectives: the technological and the educational experience. Regarding technology, mobile learning is distinguished by the use of portable equipment that allows the student to access learning objects anytime and anywhere. With regard to the educational experience, [1] defines mobile learning using keywords. In this way, mobile learning is 'personal', 'spontaneous', 'opportunistic', 'informal', 'ubiquitous', 'private', 'context sensitive', 'segmented', and 'portable'. Reference [1] also remarks that some of these features may disappear as mobile technology progress, but properties such as informality, mobility, and context will remain.

From the students' point of view, the main advantages of mobile learning are:

- The elimination of time and space constraints. Learning is available anytime and anywhere.

- It allows the communication with peers and with the teacher without physical contact.

- It allows receiving learning objects that depends on the location (i.e. context sensitive learning).

- It allows receiving instant feedback to homework and exams.

- It allows receiving learning objects that employ pictures, video, and audio.

\section{JAVA ME}

Java is an object-oriented programming language in which applications are compiled to a bytecode that runs on a virtual machine. The Java Virtual Machine (JVM) is a simulated computer that runs the bytecodes either by interpreting them or by translating them to actual machine instructions that are executed on the spot. The JVM allows programmers to write programs and run them on different operating systems without recompilation. The Java language has evolved and now consists of four editions, each of which is oriented to application development in computer equipment at a different scale. Java EE (Enterprise Edition) for servers, Java SE (Standard Edition) for personal computers, Java ME (Micro Edition) for mobile devices, and Java Card for smart cards. 
Java ME allows to design programs, called MIDlets, which can be loaded and run on Java-enabled mobile devices. The libraries available in Java ME allow to write MIDlets with graphical user interfaces and persistent storage, and permits the communication of a MIDlet with other devices or servers. However, for security, MIDlets need permission from the user to access personal information stored on the device or to communicate with other mobile equipment or with a server.

Generally, MIDlets are distributed as part of a suite, which is a set of MIDlets that perform similar tasks and share resources such as databases.

The main advantages of programming in Java ME are:

- The JVM is free and easy to install. In the case of Java-enabled cell phones and some PDAs, the JVM is already installed from factory.

- NetBeans, a development environment for Java ME that includes an editor, a compiler, and a simulator, is free.

- Reference manuals and tutorials are free.

- MIDlets are portable across different mobile operating systems.

Portability is important for mobile applications because there is no a dominant operating system such as Windows is in the personal computer market. The most popular mobile operating systems are six: Android, BlackBerry, IOS, Linux, Symbian, and Windows Mobile.

\section{RESULTS}

We have developed learning objects for descriptive Statistics by means of three suites: Module 1, Visual Sum-

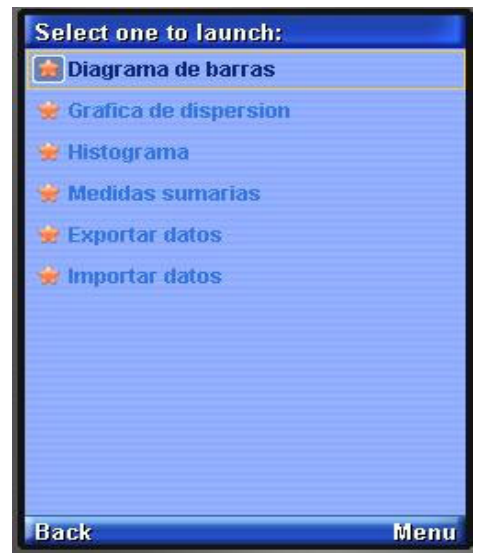

Figure 1. Suite Module 1 main menu.

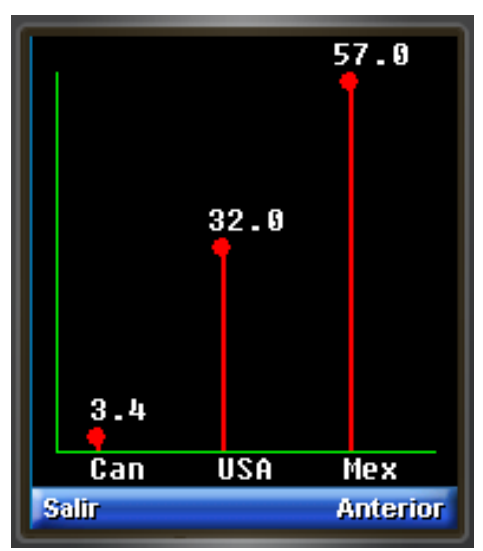

Figure 2. Bar chart. mary Measures, and Skewness Measures. We also wrote the corresponding user manual and installed the suites in five Java-enabled cell phones owned by the University of Sonora.

Now, we will describe each one of the suites.

\section{A. Module 1}

Suite Module 1 consists of six MIDlets as seen in the main menu for the suite shown in Fig. 1. Please note that all labels are in Spanish. The first four MIDlets correspond to a specific statistical tool. The last two are for exporting and importing external data files in those mobile devices that allow this functionality.

Now we will describe briefly each one of the MIDlets.

Diagrama de barras (Bar chart): This MIDlet allows to display a bar chart. The input data are written in pairs separated by spaces or line breaks (is indistinct). The first item in each pair represents a category and the second item is a number associated with that category. An example of the output of this MIDlet is shown in Fig. 2.

Diagrama de dispersión (Scatter-plot): This MIDlet allows you to display a scatter-plot, calculate the Pearson correlation index, and calculate the linear regression equation. Additionally, the MIDlet can plot the regression line on the original scatter-plot. The input data are written in pairs of numbers separated by spaces or line breaks. The pair of numbers represents the values of two variables for a set of data. Usually, the first number of the pair is the value of the independent variable, while the second number is the value of the dependent variable. Fig. 3 shows a scatter-plot with the associated regression line, while Fig. 4 presents the Pearson correlation index.

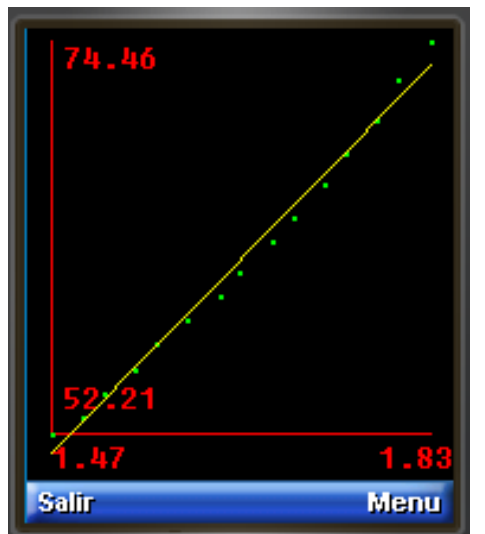

Figure 3. Scatter-plot with the regression line.

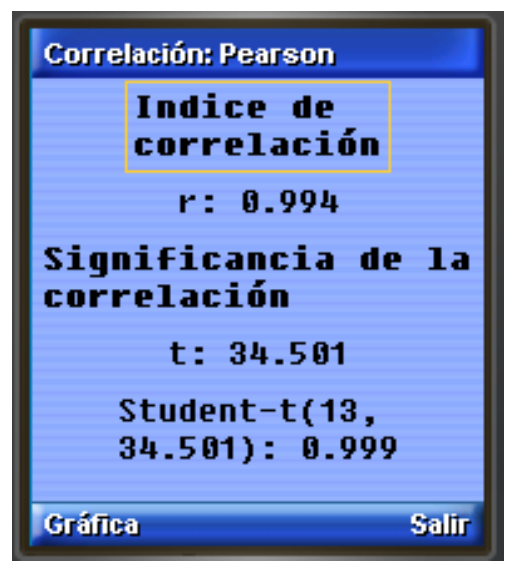

Figure 4. Pearson correlation index. 


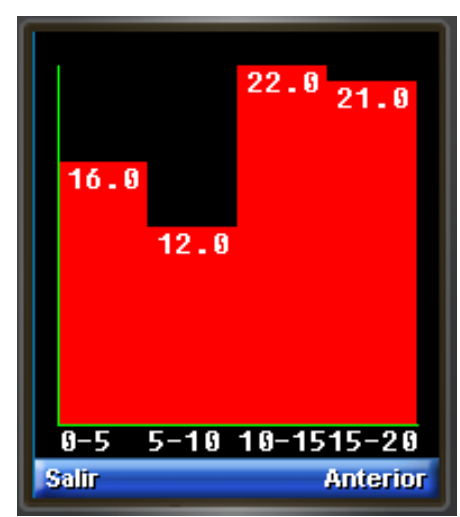

Figure 5. Histogram.

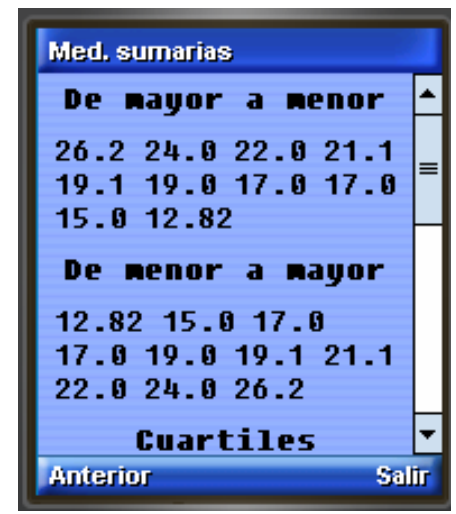

Figure 6. Summary measures.

Histograma (Histogram): This MIDlet allows to display a histogram. The input data are written in pairs of numbers separated by spaces or line breaks. The first number of the pair is the lower limit of the range and the second number is the value associated to the range. The last pair can be complete or incomplete depending on whether the latter range is open or closed. An example of the output of this MIDlet is shown in Fig. 5.

Medidas sumarias (Summary measures). This MIDlet allows to calculate several summary measures for a sequence of real numbers that are received as input. As output, the MIDlet shows the numbers sorted from highest to lowest and from lowest to highest, and the following summary measures: quartiles, deciles, mode, maximum and minimum, mean, median, standard deviation of the sample and of the population, and variance of the sample and of the population. An example of the output of this MIDlet is shown in Fig. 6.

\section{B. Visual Summary Measures}

Suite Visual Summary Measures consists of only one MIDlet which allows the user to enter a sequence of numbers and visualize up to five summary measures: mean, median, mode, standard deviation, and variance. Fig. 7 shows an example of the output of this MIDlet.

\section{Skewness Measures}

Suite Skewness Measures consists of only one MIDlet which allows the user to enter a sequence of numbers and visualize up to three measures of skewness or asymmetry: the skewness coefficients of Fisher and Pearson, and the kurtosis. For completeness, the mean, median, and mode

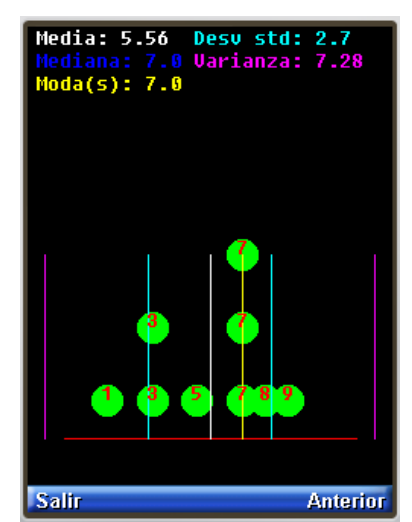

Figure 7. Visual summary measures.

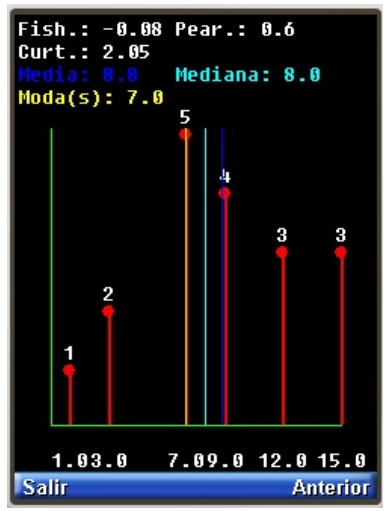

Figure 8. Skewness measures.

of the input sequence are also shown. Fig. 8 shows an example of the output of this MIDlet.

\section{LiMITATIONS}

Regarding the functionality of the suites, we have found two sources of limitations: security and computing power. The main security limitation is that there are JVM implementations that do not support optional package JSR-75, this means that the JVM does not allow a MIDlet to read or write a file external its suite. Users with such cellular phones will be required to capture data using the input method provided by the phone, typically a physical or virtual keyboard that usually is small and not very usable, instead of using the regular keyboard of a personal computer.

In relation to computing power, we have found a great variety in the existing processing capacity on mobile phones. For example, in a low-profile phone, obtaining the summary measures of a sequence of 100 numbers can take about 2 minutes, while in a medium-profile phone the response time is almost immediate.

\section{CONCLUSIONS AND FUTURE WORK}

We have presented the main achievements obtained after completing the first stage of project Statistics-to-Go. We have designed learning objects that enhance the intuitive learning of statistical concepts, exclusively using a cellular phone and taking advantage of the ubiquity of personal technology for educational purposes, specifically, the possibilities of mobile phones for teaching and learning statistics. 


\section{ELABORATION OF STATISTICS LEARNING OBJECTS FOR MOBILE DEVICES}

These learning objects cover most of the syllabus of the introductory statistics course offered in the first semesters at the University of Sonora.

As a second stage of this project, we plan to test the usability of the three suites in the students' mobile devices. That is, study the ease with which students can use their cellular phones in order to achieve a specific objective, given a learning activity of descriptive statistics. Also we will investigate the effectiveness of mobile connectivity on the university campus in order to optimize the sending and the reception of information.

\section{REFERENCES}

[1] J. Traxler, "Defining, Discussing, and Evaluating Mobile Learning: The Moving Finger Writes and Having Writ...” International Review of Research in Open and Distance Learning, vol. 8, num. 2, pp. 1-12, 2007.

\section{AUTHORS}

F. J. Tapia-Moreno is with the Department of Mathematics, University of Sonora, Hermosillo, Sonora 83000 Mexico (e-mail: ftapia@gauss.mat.uson.mx).

H. A. Villa-Martínez is with the Department of Mathematics, University of Sonora, Hermosillo, Sonora 83000 Mexico (e-mail: hvilla@gauss.mat.uson.mx).

C. A. Lopez-Miranda is with the Department of Mathematics, University of Sonora, Hermosillo, Sonora 83000 Mexico (e-mail: claudio@gauss.mat.uson.mx)

This work was supported in part by a PIFI fund from the Mexican federal government. Received 2 February 2012. Published as resubmitted by the authors on March, 30, 2012. 